\title{
SU(2) cosmological solitons
}

\author{
C. Lechner \\ Institute for Theoretical Physics, University of Vienna, Boltzmanng. 5 A-1090 Wien, Austria
}

S. Husa

Department of Physics and Astronomy, University of Pittsburgh, Pittsburgh, Pennsylvania 15260 and Max-Planck-Institute for Gravitational Physics, Albert Einstein Institute, Am Mühlenberg 1, D-14476 Golm, Germany

\author{
P. C. Aichelburg \\ Institute for Theoretical Physics, University of Vienna, Boltzmanng. 5 A-1090 Wien, Austria
}

(Received 8 February 2000; published 27 July 2000)

\begin{abstract}
We present a class of numerical solutions to the SU(2) nonlinear $\sigma$ model coupled to the Einstein equations with a cosmological constant $\Lambda \geqslant 0$ in spherical symmetry. These solutions are characterized by the presence of a regular static region which includes a center of symmetry. They are parametrized by a dimensionless “coupling constant" $\beta$, the sign of the cosmological constant, and an integer "excitation number" $n$. The phenomenology we find is compared to the corresponding solutions found for the Einstein-Yang-Mills (EYM) equations with a positive $\Lambda$ (EYM $\Lambda$ ). If we choose $\Lambda$ positive and fix $n$, we find a family of static spacetimes with a Killing horizon for $0 \leqslant \beta<\beta_{\max }$. As a limiting solution for $\beta=\beta_{\max }$ we find a globally static spacetime with $\Lambda=0$, the lowest excitation being the Einstein static universe. To interpret the physical significance of the Killing horizon in the cosmological context, we apply the concept of a trapping horizon as formulated by Hayward. For small values of $\beta$ an asymptotically de Sitter dynamic region contains the static region within a Killing horizon of cosmological type. For strong coupling the static region contains an "eternal cosmological black hole.',

PACS number(s): 04.40.-b, 04.25.Dm, 04.70.Bw, 98.80.Hw
\end{abstract}

\section{INTRODUCTION}

The aim of this paper is to discuss the static spherically symmetric solutions of the $\mathrm{SU}(2)$ nonlinear $\sigma$ model coupled to the Einstein equations with a cosmological constant $\Lambda$ $\geqslant 0$ (subsequently referred to as the $\mathrm{E} \sigma_{\mathrm{SU}(2)} \Lambda$ model). The existence of such solutions was suggested by the fact that the Einstein universe [3] and the de Sitter spacetime [4] admit the existence of a discrete one-parameter family of static, spherically symmetric regular solutions of the $\mathrm{SU}(2)-\sigma$ model. These solutions are unstable, the number of unstable modes equals the "excitation index." The static solutions on the de Sitter background correspond to the uncoupled limit, i.e., the vanishing coupling constant of the $\mathrm{E} \sigma_{\mathrm{SU}(2)} \Lambda$ model.

Globally regular stationary "solitonic'" solutions, if they exist, play a fundamental role in the dynamics of gravitating systems. If they are stable, they provide possible end states of evolution-and models for "stars" or " "particles." Interest in solitonic solutions and the subtleties of the interaction of matter with gravity was revived by the surprising numerical discovery of static solutions to the Einstein-Yang-Mills (EYM) system by Bartnik and McKinnon [5]. It was soon found that these solutions are unstable [6], but there is a link between this instability and another surprise in general relativity (GR):

In recent years the discovery of critical phenomena at the threshold of black hole formation has introduced a new twist into the gravitational collapse problem. In the original work of Choptuik the intermediate attractor that separates the two possible generic end-states of a free scalar field-the formation of a black hole and complete dispersion-is the self- similar "choptuon", which forms a naked singularity. However, as was discovered by Choptuik, Chmaj, and Bizon [7], also unstable solitons can serve as an intermediate attractor in critical collapse. In particular they found that the first Bartnik-McKinnon excitation actually forms an intermediate attractor associated with type I critical collapse phenomena - as opposed to the type II originally found by Choptuik (both named because of the correspondence to phenomena of statistical physics, for a recent overview on critical collapse phenomena see, e.g., Choptuik [8]).

While both the free scalar field and the EYM system do not contain any dimensionless parameters, the $\sigma$ models are simple systems with a dimensionless coupling constant. Thus they provide a convenient family of theories that is suitable to study genericity and bifurcation phenomena.

It is known that, apart from trivial solutions, the nonlinear $\sigma$-models do not admit soliton solutions in Minkowski space, and when coupled to gravity, there exist neither solitons nor static black hole solutions that are asymptotically flat (see, e.g., Ref. [9]). The presence of a positive cosmological constant changes this situation by introducing a length scale into the model. From dimensional analysis one concludes that the behavior of the solutions depends nonperturbatively on $\Lambda$, and that only the sign of $\Lambda$ is significant. Within the static spacetimes we restrict ourselves to spacetimes which possess a static region that has a regular center and is bounded by at most one Killing horizon (as opposed to two, such as in the Schwarzschild-de Sitter case).

In the coupled case we find four qualitatively different types of solutions along each "branch" defined by the "excitation index." For small coupling constant of the $\sigma$ model, 
the solutions are globally regular, and a Killing horizon separates a static region from an asymptotically de Sitter dynamic region. For intermediate values of the coupling constant the situation is similar, but the region exterior to the cosmological horizon expands for some time, but eventually recollapses. For even higher values of the coupling constant the horizon encloses a dynamic region that undergoes collapse. Thus in some sense the static region gets turned "inside out."'

Solutions with a finite regular static region and positive cosmological constant only exist for a coupling constant smaller than some critical value which depends on the excitation index. The limit is singular, but combining it with a limit $\Lambda \rightarrow 0$ yields solutions which are globally regular and static and have spatial topology $S^{3}$.

For $\Lambda>0$ the "first static excited states" exhibit one unstable mode, so that our solutions form a one parameterfamily of candidate solutions for type I intermediate attractors. The full linear stability analysis, that leads to this result, will be presented in a separate paper [10]. Similar results have been found for the EYM $\Lambda$ system, which has been studied in great detail by Volkov et al. [1].

The organization of this paper is as follows. In Sec. II we introduce our nonlinear $\sigma$ model and discuss its basic properties. We then specialize to spherical symmetry using the hedgehog ansatz for the SU(2)-valued matter field and write out the spherically symmetric field equations in a suitable gauge.

The problem of finding regular static solutions of the $\mathrm{E} \sigma_{\mathrm{SU}(2)} \Lambda$ problem is discussed in Sec. III. The static regions containing the soliton are confined within a Killing horizon, outside of which the timelike Killing vector becomes spacelike and the spacetimes are dynamical. The static regions are constructed by solving a boundary value problem as described in Sec. III D. The global structure of the spacetimes is then determined by evolving the static data beyond the horizon. This evolution problem is discussed in Sec. III E. The phenomenology of the solutions we find is presented in Sec. IV. Finally, Sec. V gives a discussion of our results and compares them to the results of Volkov et al. [1] for the EYM $\Lambda$ system.

Conventions are chosen as follows. Spacetime indices are greek letters, $\mathrm{SU}(2)$ indices are uppercase latin letters, the spacetime signature is $(-,+,+,+)$, the Ricci tensor is defined as $\mathcal{R}_{\mu \nu}=\mathcal{R}_{\mu \lambda \nu}^{\lambda}$, and the speed of light is set to unity, $c=1$.

\section{THE SU(2)- $\sigma$ MODEL IN SPHERICAL SYMMETRY}

\section{A. Harmonic maps as matter models}

Nonlinear $\sigma$ models are special cases of harmonic maps from a spacetime $\left(\mathbf{M}, g_{\mu \nu}\right)$ into some target manifold $\left(\mathbf{N}, G_{A B}\right)$ (see e.g., Ref. [11]). For the $\mathrm{SU}(2)-\sigma$ model, the target manifold is taken as $S^{3}$ with $G_{A B}$ the "round" metric of constant curvature. Harmonic maps $X^{A}\left(x^{\mu}\right)$ are extrema of the simple geometric action

$$
S=-\frac{1}{2} \gamma \int_{\mathbf{M}} d^{m} x \sqrt{|g|} g^{\mu \nu} \partial_{\mu} X^{A} \partial_{\nu} X^{B} G_{A B}(X) .
$$

Their importance for physics was pointed out in a review article by Misner [12].

Variation of the matter action (2.1) with respect to the metric $g_{\mu \nu}$ yields the stress-energy tensor of the harmonic map

$$
\begin{aligned}
T_{\mu \nu}= & \gamma\left(\nabla_{\mu} X^{A} \nabla_{\nu} X^{B} G_{A B}\left(X^{C}\right)\right. \\
& \left.-\frac{1}{2} g_{\mu \nu} \nabla^{\sigma} X^{A} \nabla_{\sigma} X^{B} G_{A B}\left(X^{C}\right)\right) .
\end{aligned}
$$

This stress-energy-tensor obeys the weak, strong, and dominant energy conditions.

In order to produce static configurations attractive and repulsive forces have to be balanced. In particular in flat space the virial theorem implies that the components of the stress-energy tensor cannot have a fixed sign (see the discussion by Gibbons [13]). For static configurations of any harmonic map the sum of the principal pressures $\sum_{\hat{i}=1}^{3} T_{\hat{i} \hat{i}}$ is nonpositive everywhere [13] ( $T_{\hat{i} \hat{j}}$ denote the spatial components of the stress-energy tensor with respect to an orthonormal frame). The self-interaction of any $\sigma$ model can therefore be interpreted as attractive. In contrast for the Yang Mills field the sum of the principal pressures is nonnegative, which can be interpreted as a repulsive self-interaction. Both fields therefore do not allow soliton solutions on a flat background. But while the Yang-Mills field coupled to gravity admits solitons by cancelling the repulsive force with gravity [14], the gravitating $\sigma$ model correspondingly does not (see, e.g., Ref. [9]).

Thus we cannot expect static solutions to exist unless we add some "repulsive force" - or in the presence of nontrivial topology. As an example for the latter one could think of a static spherical universe of topology $S^{3}$, where there is a balance between the tendencies to collapse towards either "center." A repulsive force on the other hand can be introduced with a positive cosmological constant $\Lambda$. Therefore we consider the total action

$$
S=\int d^{4} x \sqrt{-g}\left(\frac{1}{16 \pi G}(\mathcal{R}-2 \Lambda)+\mathcal{L}_{M}\right),
$$

where $\mathcal{L}_{M}$ is given by the Lagrangian of Eq. (2.1). This action gives rise to the Einstein equations

$$
\mathcal{R}_{\mu \nu}-\frac{1}{2} g_{\mu \nu} \mathcal{R}+\Lambda g_{\mu \nu}=8 \pi G T_{\mu \nu},
$$

as well as to the field equations of the harmonic map

$$
g^{\mu \nu}\left[\nabla_{\mu} \nabla_{\nu} X^{A}+\Gamma_{B C}^{A}\left(X^{D}\right) \nabla_{\mu} X^{B} \nabla_{\nu} X^{C}\right]=0,
$$

where $\widetilde{\Gamma}_{B C}^{A}$ denote the Christoffel symbols with respect to $G_{A B}$. The field equations thus allow a general nonlinear dependence on the fields $X^{A}$ through the Christoffel symbols and they depend quadratically on the first derivatives of the fields. 
In units where $c=1$ the coupling constant $\gamma$ of the harmonic map has dimension mass/length, whereas the gravitational constant $G$ is of dimension length/mass. Both constants enter the equations only in the dimensionless product $\beta=4 \pi G \gamma$, thereby defining a one-parameter family of distinct gravitating matter models. The parameter $\Lambda$ plays a different role, since it has dimension $1 /$ length $^{2}$. Thus, when the cosmological constant is nonzero, it provides the length scale of these theories. Therefore all theories with the same value of $\beta$ are equivalent irrespective of the value of $\Lambda$. If, on the other hand, $\Lambda=0$, the field equations are scale invariant. The gravitating $\mathrm{SU}(2)-\sigma$ model with non-negative cosmological constant thus corresponds to a two-parameter family of inequivalent theories, parametrized by the continuous parameter $\beta$ and the discrete parameter $\operatorname{sgn}(\Lambda)$.

\section{B. Spherical symmetry}

A spherically symmetric metric can be written in the general form as the warped product (see, e.g., Ref. [15])

$$
d s^{2}=d \tau^{2}+R^{2}(\rho, t) d \Omega^{2},
$$

where $d \tau^{2}$ is a general two-dimensional Lorentzian line element and the area of the orbits of $\mathrm{SO}(3)$ is given by $4 \pi R^{2}(\rho, t)$. Choosing the coordinates $t$ and $\rho$ orthogonal one can write the metric in the form

$$
d s^{2}=-A(\rho, t) d t^{2}+B(\rho, t) d \rho^{2}+R^{2}(\rho, t) d \Omega^{2} .
$$

In Eq. (2.7) there is some gauge freedom left, which can be used to eliminate one of the three functions $A, B$, or $R$.

One way to fix the gauge would be to use the function $R$ as a coordinate. This is possible as long as $\nabla_{\mu} R \neq 0$. This gauge is usually referred to as the Schwarzschild gauge. Here we will deal with the situation that $\nabla_{\mu} R$ becomes zero on some maximal two sphere (which is not a horizon), a phenomenon which we will discuss in detail below.

Following Volkov et al. [1] we therefore choose a different gauge, which keeps close to the standard Schwarzschild line element in another way: we fix $A(\rho, t)=1 / B(\rho, t)$ $\equiv Q(\rho, t)$ and keep the area of the $\mathrm{SO}(3)$ orbits as the second free function

$$
d s^{2}=-Q(\rho, t) d t^{2}+\frac{1}{Q(\rho, t)} d \rho^{2}+R^{2}(\rho, t) d \Omega^{2} .
$$

The metric (2.8) is regular where $Q(\rho, t)$ and $R(\rho, t)$ are regular functions on spacetime, except possibly at points where either $Q(\rho, t)$ or $R(\rho, t)$ vanish. The vanishing of $Q(\rho, t)$ is related to the existence of horizons, which will also be discussed below.

For a regular spacetime the vanishing of $R(\rho, t)$ is associated with the singularity of spherical coordinates at the axis of symmetry. Such a coordinate center does not necessarily have to be present (e.g., in a spherical wormhole), but we will require our spacetimes to possess at least one regular center-there may also be two, such as in the Einstein static universe written in spherical coordinates. In the following, several boundary conditions will be derived from regularity requirements, we will therefore make some comments on regularity near the center of spherical symmetry for the metric defined in Eq. (2.8): We assume the existence of four regular (meaning $C^{\infty}$ or $C^{k}$ as appropriate) coordinate functions $x, y, z, t$ on the manifold. All other functions are defined as regular if they can be expressed as regular functions of $x$, $y, z, t$. Furthermore, the relations between the functions $R, \theta$, $\phi$ and $x, y, z$ are required to be the standard coordinate transformation between Cartesian and spherical coordinates: $x$ $=R \sin \phi \sin \theta, y=R \cos \phi \sin \theta, z=R \cos \theta$. Note that the spherical coordinates, in particular $R$, are not regular functions, but all even powers of $R$ are. Near the axis we choose the parametrization of the radial coordinate $\rho$ such that it has the same regularity features as $R$ :

$$
\rho=R h\left(R^{2}\right),
$$

where $h$ is a regular positive function - and thus also $\rho$ itself is not regular. Any spherically symmetric function which is a regular function of $x, y, z, t$ (or just "regular" for short) can therefore be written as a regular function of $\rho^{2}$ and $t$.

For any fixed $t$ the center $R=0$ then is a regular point of spacetime, if $Q(0, t)=h(0)$. Without restricting generality we can choose $h(0)=1$ for convenience, such that

$$
\begin{aligned}
& R(\rho, t)=\rho\left(1+O\left(\rho^{2}\right)\right), \\
& Q(\rho, t)=1+O\left(\rho^{2}\right) .
\end{aligned}
$$

In spherical symmetry it is possible to define a quasilocal mass

$$
m=\frac{R}{2}\left(1-\nabla_{\mu} R \nabla^{\mu} R\right)
$$

which reads

$$
m=\frac{R}{2}\left(1-\frac{\dot{R}^{2}}{Q}-Q\left(R^{\prime}\right)^{2}\right) .
$$

in our coordinate system (2.8). For a recent discussion of the properties of the quasilocal mass in spherical symmetry see Hayward [16].

The harmonic map field configuration $X^{A}\left(x^{\mu}\right)$ can be called spherically symmetric, if the Lie derivatives of the energy-momentum tensor with respect to the Killing vector fields that generate the $\mathrm{SO}(3)$ action vanish. One possibility to achieve this, is to demand that all fields be functions of $\rho$ and $t$ only. This would leave us with a coupled system of differential equations for three fields and two metric functions. Since the target manifold $\left(S^{3}, G\right)$ also admits $\mathrm{SO}(3)$ as an isometry group, there is an alternative way to impose spherical symmetry on the harmonic map, which is the well known hedgehog ansatz. We first introduce spherical coordinates $(f, \Theta, \Phi)$ on the target manifold, writing the $\mathrm{SU}(2)$ line element as

$$
d s^{2}=d f^{2}+\sin ^{2} f\left(d \Theta^{2}+\sin ^{2} \Theta d \Phi^{2}\right) .
$$


The hedgehog ansatz now ties the coordinates on the target manifold to those on the base manifold:

$$
f\left(x^{\mu}\right)=f(\rho, t), \quad \Theta\left(x^{\mu}\right)=\theta, \quad \Phi\left(x^{\mu}\right)=\varphi .
$$

Due to this ansatz two of the three coupled fields are already determined and only one field $f(\rho, t)$ enters the equations. The matter field equations (2.5) are then reduced to the single nonlinear wave equation

$$
\square f=\frac{\sin (2 f)}{R^{2}},
$$

where $\square$ is the wave operator of the spacetime metric.

Regularity of the harmonic map within this ansatz means that when expressing the field in terms of regular coordinates $X^{A}$ on SU(2), the $X^{A}$ are regular fields on spacetime. Since $X^{A}\left(x^{\mu}\right)=[f(\rho, t) / \rho] x^{A}$, where $x^{A}$ denote the Cartesian coordinates $x, y, z$ on spacetime, the field $f(\rho, t)$ thus has to be of the form

$$
f(\rho, t)=\rho H\left(\rho^{2}\right)=f^{\prime}(0) \rho+O\left(\rho^{3}\right),
$$

where $H$ is a regular function. Thus $f$ vanishes at the center, which means that the center $R=0$ is mapped to one of the poles of the target manifold, that have been fixed via the hedgehog ansatz.

\section{STATIC SOLUTIONS}

\section{A. Static field equations}

In addition to spherical symmetry we assume that spacetime admits a hypersurface orthogonal Killing vector field $\partial_{t}$, which is timelike in some neighborhood of the (regular) center $R=0$. The combinations $\left(\begin{array}{c}t \\ t\end{array}\right)-\left(\begin{array}{c}\rho \\ \rho\end{array}\right)-2\left(\begin{array}{c}\theta \\ \theta\end{array}\right)$ and $\left(\begin{array}{c}t \\ t\end{array}\right)-\left(\begin{array}{c}\rho \\ \rho\end{array}\right)$ of the mixed components of Einstein's equations plus the matter field equation (2.14) then yield the following set of coupled second order autonomous ODEs for the metric functions $Q(\rho), R(\rho)$ and the matter field $f(\rho)$ :

$$
\begin{aligned}
\left(R^{2} Q^{\prime}\right)^{\prime} & =-2 \Lambda R^{2}, \\
R^{\prime \prime} & =-\beta R f^{\prime 2}, \\
\left(Q R^{2} f^{\prime}\right)^{\prime} & =\sin 2 f .
\end{aligned}
$$

Furthermore the above system of equations has a constant of motion:

$$
2 \beta \sin ^{2} f+R^{2}\left(\Lambda-\beta Q f^{\prime 2}\right)+R Q^{\prime} R^{\prime}+Q R^{\prime 2}-1=0 .
$$

This expression can either be derived by integrating the system (3.1)-(3.3) and using the regularity conditions at the axis, or by using the $\left({ }_{\rho}^{\rho}\right)$ component of Einstein's equations. Note that if $f$ is a matter field solution, then so is also $k \pi$ $\pm f$ for any integer $k$.

If $\Lambda$ is nonzero it sets the length scale, it thus can be eliminated in these equations by using the dimensionless quantities $\bar{\rho}=\sqrt{\Lambda} \rho$ and $\bar{R}=\sqrt{\Lambda} R$. If, on the other hand, $\Lambda$ vanishes, the equations are scale invariant, and thus invariant under rescalings $\bar{\rho}=a \rho$ and $\bar{R}=a R$. Furthermore, in this case Eq. (3.1) together with regularity conditions at the axis implies $Q \equiv 1$.

If we integrate the equations with the above boundary conditions from $\rho=0$ to larger values of $\rho$ one of the following four situations has to occur: (1) the static region "ends" in a singularity; (2) integration might run into a second (regular) pole $R=0$, which would mean that the resulting spacetime has compact slices of constant $t$ and is globally static; (3) the static region might persist up to spatial infinity; (4) the static region might be surrounded by a Killing horizon beyond which spacetime becomes dynamical. The first case can easily be produced by shooting off a regular center with arbitrary initial data, it can be excluded by setting up a boundary value problem that enforces one of the other three cases. The second case can be discarded for our model with positive cosmological constant, since the existence of a static region with two regular centers is not compatible with the field equations: To see this recast Eq. (3.1) into the integral form (3.21). It is clear then, that-for nonzero $\Lambda-Q^{\prime}$ diverges if $R$ goes to zero a second time. For $\Lambda=0$ of course, such solutions may exist as, e.g., the static Einstein universe (3.7), which will be discussed in the next section. The third case can also be excluded if the cosmological constant is positive: a static region can not be extended to spatial infinity, but rather a singular point of the equations $Q\left(\rho_{H}\right)=0$, that corresponds to a horizon, has to develop. This can be seen as follows: Suppose the solution exists up to $\rho \rightarrow \infty$ and $R$ is monotonically increasing - all other assumptions automatically lead to one of the cases 1,2 , or 4-then one can show, that for $\Lambda$ positive $Q^{\prime}<$ - const $/ \rho$ for $\rho$ large enough, which means that $Q$ would be bounded from above by a function that tends to $-\infty$ as $\rho$ $\rightarrow \infty$. So again $Q$ has to cross zero at some finite value of $\rho$.

For positive cosmological constant we therefore may confine ourselves to the cases with horizon, and we will construct static regions as an ordinary differential equation (ODE) boundary value problem, where the boundary conditions correspond to a regular center at $\rho=0$ and a regular horizon at $\rho=\rho_{H}$, where the determination of the value of $\rho_{H}$ is part of the boundary value problem. The appropriate boundary conditions at the horizon will be determined in Sec. III C, and the boundary value problem is described in Sec. III D.

Given a static region, i.e., a region of spacetime where the Killing vector $\partial_{t}$ is timelike, which is bounded by a Killing horizon, we can consider as a second step the time evolution problem of the static data on the horizon into the dynamic region where the Killing vector $\partial_{t}$ is spacelike and the spacetime is thus homogeneous. The time evolution problem thus reduces to a system of ODEs which are solved as an initial value problem as described in Sec. III E.

\section{B. Exact solutions}

Some solutions of Eqs. (3.1)-(3.4) with a regular center can be given in closed form. They do arise as certain limits of the numerically constructed family of solutions to be given below. 
First of all, for $\Lambda>0$, for the trivial case $f \equiv 0$ Eq. (3.2) has the solutions $R(\rho)=a \rho+b$. Imposing the regularity conditions (2.9) this gives de Sitter spacetime

$$
R(\rho)=\rho, \quad Q(\rho)=1-\frac{\Lambda \rho^{2}}{3}, \quad f \equiv 0 .
$$

For $f \equiv \pi / 2, R(\rho)$ is of the same form as above. For $b$ $=0$ we get

$$
R(\rho)=\rho, \quad Q(\rho)=1-2 \beta-\frac{\Lambda \rho^{2}}{3}, \quad f \equiv \frac{\pi}{2},
$$

which looks similar to de Sitter for large $\rho$ but has a conical singularity at the center if $\beta>0$. Furthermore the static region shrinks with increasing $\beta$ and ceases to exist for $\beta$ $=1 / 2$. In the limit of vanishing coupling constant $\beta=0$, where spacetime is de Sitter, this solution $f \equiv \pi / 2$ is still singular at the center with diverging energy density. Nevertheless the total energy is finite, and this solution may be viewed as the "high excitation" limit of the regular solutions, that exist on de Sitter background [4].

Finally, for $\Lambda=0$ Eq. (3.1), together with regularity conditions at the axis, yields $Q \equiv 1$. For $\beta=1$ the remaining equations can be solved analytically to give the static Einstein universe

$$
R(\rho)=\sin \rho, \quad Q(\rho) \equiv 1, \quad f(\rho)=\rho .
$$

Note that the stress-energy tensor, which has the form of a perfect fluid in this case satisfies $\mu+3 p=0$. As will be described in detail in Sec. IV C the static Einstein universe arises in the limit of maximal coupling constant of the numerical constructed first excitation if at the same time $\Lambda$ is set to zero.

For completeness we mention that there also exist exact solutions to Eqs. (3.1)-(3.4), that do not possess a regular center of spherical symmetry, such as the Nariai spacetime.

\section{Horizons and global structure}

In order to discuss the global structure of the spacetime and in particular regularity questions from which we derive the boundary conditions for our ODEs, it is helpful to consider a coordinate system, which is regular at the horizon. We write the metric (2.8) as

$$
d s^{2}=-Q(\rho) d u^{2}-2 d u d \rho+R(\rho)^{2} d \Omega^{2},
$$

where the coordinate function $\rho$ and the metric function $Q(\rho)$ coincide with those in the metric (2.8), and the coordinate $u$ is given as

$$
u=t-\int \frac{d \rho}{Q(\rho)} .
$$

Note that the coordinates (3.8) cover only half of the maximally extended spacetime. In the following, we will simplify our discussion by only talking about the Killing horizon contained in the portion of spacetime covered here. All statements made can be extended trivially to the com- plete spacetime and in particular the second component of the horizon by time reflection. We also remark that all solutions have the topology $S^{3} \times R$.

The static Killing vector field is $\partial / \partial u=\partial / \partial t$, where the latter is taken with respect to the $(t, \rho)$ coordinates. The metric (3.8) is regular if $Q(\rho)$ and $R(\rho)$ are regular functions, except when $R=0$, which corresponds either to the usual coordinate singularity of spherical symmetry, which has been discussed in Sec. II B or to a spacetime singularity, as discussed in Sec. III E.

The Killing vector field $\partial_{u}$, which we have assumed to be timelike in some neighborhood of the (regular) center $R=0$ need not necessarily be globally timelike. Regions where it becomes spacelike, i.e., $Q(\rho)<0$, are dynamic with homogeneous spacelike slices of constant time $\rho$, the spatial topology being $S^{2} \times R$. Such regions thus correspond to Kantowski-Sachs models. At the boundary of static and dynamic regions the metric function $Q(\rho)$ vanishes, such a surface of $\rho=$ const is thus a null surface. Furthermore the Killing vector field $\partial_{u}$ is null and tangent on this surface, which therefore is a Killing horizon.

In the presence of an asymptotic infinity, such as in an asymptotically flat or asymptotically de Sitter spacetime, one can use the asymptotic region to classify event horizons, e.g., as black hole or cosmological event horizons. Furthermore one is provided a straightforward definition of "inward" and "outward" directions. In the cosmological case these issues are less clear. We therefore follow the definitions of Hayward [2]: He proposed a local definition of a trapping horizon, a concept which does not make use of asymptotic flatness and is therefore also suitable for more general situations. Intuitively, the physical interpretation of the Killing horizon depends on whether the dynamical region is collapsing or expanding off the horizon, and whether this region is to be interpreted as inside or outside. Both can be formulated in terms of the null expansions $\Theta_{ \pm}$of ingoing and outgoing null rays from two surfaces. In spherical symmetry we consider $R=$ const surfaces, with null expansions

$$
\Theta_{ \pm}=\frac{1}{R^{2}} \mathcal{L}_{ \pm} R^{2},
$$

where $\mathcal{L}_{ \pm}$is the Lie-derivative along the null directions

$$
l_{+}=\partial_{\rho} \quad \text { and } \quad l_{-}=2 \partial_{u}-Q \partial_{\rho},
$$

respectively, so

$$
\Theta_{+}=2 \frac{R^{\prime}}{R} \quad \text { and } \Theta_{-}=-2 Q \frac{R^{\prime}}{R} .
$$

The first use of the expansions is to define a trapped surface in the sense of Penrose [17] as a compact spatial surface for which $\Theta_{-} \Theta_{+}>0$. If one of the expansions vanishes, the surface is called a marginal surface. For a nontrapped surface $\Theta_{-}$and $\Theta_{+}$have opposite signs, and we call directions in which the expansion is positive outward, and inward when it is negative. 
On the Killing horizon $Q=0,\left.\quad \Theta_{-}\right|_{Q=0}=0$ while $\left.\Theta_{+}\right|_{Q=0} \neq 0$ and $\left.\mathcal{L}_{+} \Theta_{-}\right|_{Q=0} \neq 0$ (except when also $R^{\prime}=0$, which we exclude for the moment). In Hayward's terminology [2] such a three-surface is called a trapping horizon. It is said to be outer if $\left.\mathcal{L}_{+} \Theta_{-}\right|_{Q=0}<0$, inner if $\left.\mathcal{L}_{+} \Theta_{-}\right|_{Q=0}<0$, future if $\left.\Theta_{+}\right|_{Q=0}<0$, and past if $\left.\Theta_{+}\right|_{Q=0}>0$. A future outer trapping horizon provides a general definition of a black hole, while a white hole has a past outer horizon, and cosmological horizons are inner horizons.

If $R^{\prime}(\rho)=0$ within the static region then

$$
\left.\mathcal{L}_{+} \Theta_{-}\right|_{Q=0}<0,\left.\quad \Theta_{+}\right|_{Q=0}<0
$$

and the surface $Q=0$ is a future outer trapping horizon. On the other hand if $R^{\prime}$ vanishes in the dynamical region or is monotonic, then the signs in Eq. (3.13) are reversed and one speaks of a past inner trapping horizon.

Note, that at points where $R^{\prime}=0$, both $\Theta_{ \pm}=0$. However, this does not lead to a (trapping) horizon since the expansions merely change sign. The significance of such marginal surfaces is that the meaning of inward and outward directions are reversed. A typical example is an "equatorial" two surface of a round three sphere at a moment of time symmetry.

Using the expansions $\Theta_{+}$and $\Theta_{-}$(3.12) one can rewrite the quasilocal mass $(2.11)$

$$
m=\frac{R}{2}\left(1+\frac{R^{2}}{4} \Theta_{+} \Theta_{-}\right) .
$$

Using the above assignment of inward and outward direction the quasilocal mass can be interpreted as the total mass that is contained within any spatial three volume, that is bounded by the two-sphere with areal radius $R$. On the marginal surfaces, where $R^{\prime}=0$, the quasilocal mass equals $R / 2$.

\section{The boundary value problem for the static region}

The system of ODEs (3.1)-(3.3) has singular points for $R=0$ and $Q=0$. Making use of the imposed regularity conditions at the axis (2.9) and (2.15) the equations demand in addition $Q^{\prime \prime}(0)=-2 \Lambda / 3$. Note that solutions, that are regular in a neighborhood of $\rho=0$ are determined by the value of the single parameter $c \equiv f^{\prime}(0)$.

The other singular point occurs at the horizon, where $Q\left(\rho_{H}\right)=0$. Formal Taylor series expansions around $\rho=\rho_{H}$ give

$$
\begin{aligned}
Q(\rho)= & Q_{H}^{\prime}\left(\rho-\rho_{H}\right)+\frac{Q^{\prime \prime}\left(\rho_{H}\right)}{2}\left(\rho-\rho_{H}\right)^{2}+O\left[\left(\rho-\rho_{H}\right)^{3}\right] \\
R(\rho)= & R_{H}+R^{\prime}\left(\rho_{H}\right)\left(\rho-\rho_{H}\right)+\frac{R^{\prime \prime}\left(\rho_{H}\right)}{2}\left(\rho-\rho_{H}\right)^{2} \\
& +O\left[\left(\rho-\rho_{H}\right)^{3}\right] \\
& \\
f(\rho)= & b+f^{\prime}\left(\rho_{H}\right)\left(\rho-\rho_{H}\right)+\frac{f^{\prime \prime}\left(\rho_{H}\right)}{2}\left(\rho-\rho_{H}\right)^{2} \\
& +O\left[\left(\rho-\rho_{H}\right)^{3}\right]
\end{aligned}
$$

where

$$
\rho_{H}, \quad f\left(\rho_{H}\right)=b, \quad R\left(\rho_{H}\right)=R_{H}, \quad Q^{\prime}\left(\rho_{H}\right)=Q_{H}^{\prime}
$$

are free shooting parameters. The other coefficients are determined by the requirement that $Q$ and $R$ be regular functions of $\rho$. Consistency with Eqs. (3.1)-(3.4) then amounts to the conditions

$$
\begin{aligned}
R^{\prime}\left(\rho_{H}\right) & =\frac{1-2 \beta \sin ^{2} b-R_{H}^{2} \Lambda}{R_{H} Q_{H}^{\prime}} \\
Q^{\prime \prime}\left(\rho_{H}\right) & =-2 \frac{R^{\prime}\left(\rho_{H}\right) Q^{\prime}\left(\rho_{H}\right)}{R_{H}}-2 \Lambda \\
f^{\prime}\left(\rho_{H}\right) & =\frac{\sin 2 b}{Q^{\prime}\left(\rho_{H}\right) R_{H}^{2}}, \\
f^{\prime \prime}\left(\rho_{H}\right) & =\frac{\sin 2 b}{Q^{\prime}\left(\rho_{H}\right) R_{H}^{2}}\left(-\frac{R^{\prime}\left(\rho_{H}\right)}{R_{H}}+\cos 2 b+\frac{\Lambda}{Q^{\prime}\left(\rho_{H}\right)}\right) .
\end{aligned}
$$

In order to determine the spectrum of static solutions for a fixed coupling constant $\beta$, we solve the boundary value problem Eqs. (3.1) -(3.3) with boundary conditions (2.9), (2.15), and (3.15) between axis and horizon. We use a standard two point shooting and matching method (routine D02AGF of the NAG library [18]), where the parameters $f^{\prime}(0), \rho_{H}, f\left(\rho_{H}\right), R_{H}$, and $Q_{H}^{\prime}$ serve as shooting parameters.

For $\beta=0$ a discrete one-parameter family of solutions has already been discussed in [4]. In order to get good initial guesses for the shooting parameters for $\beta>0$, we follow one solution from $\beta=0$ up to higher values of $\beta$, interpolating the values of the shooting parameters at the present and last " $\beta$ step", to obtain values for the next " $\beta$ step."

\section{E. Integration through the horizon}

Since the singularity at the horizon is merely a coordinate singularity, we can extend spacetime through the horizon and reintroduce the coordinates in Eq. (2.8) in the dynamic region beyond the horizon. The Killing vector field $\partial_{t}$ becomes spacelike, and instead of $t$ the timelike coordinate is $\rho$. The coupled system of ODEs (3.1)-(3.3) together with initial conditions (3.15) therefore constitute an initial value problem.

While integrating forward in time $\rho$, essentially two things can happen according to the behavior of $R(\rho)$.

(1) $R(\rho)$ is monotonically increasing for all $\rho>0$. Then time evolution exists for all $\rho>0$. This can be seen by turning Eqs. (3.1)-(3.3) into integral equations

$$
Q^{\prime}=-\frac{2 \Lambda}{R^{2}} \int_{0}^{\rho} R^{2} d \bar{\rho},
$$




$$
\begin{aligned}
& R^{\prime}=1-\beta \int_{0}^{\rho} R f^{\prime 2} d \bar{\rho}, \\
& f^{\prime}=\frac{1}{Q R^{2}} \int_{0}^{\rho} \sin (2 f) d \bar{\rho} .
\end{aligned}
$$

All first derivatives are bounded as long as $Q$ and $R$ do not go to zero. Beyond the horizon $Q$ cannot go to zero, since $Q^{\prime}<0$ for all $\rho>0$ and $Q\left(\rho_{H}\right)=0 . R$ cannot go to zero since it is monotonically increasing by assumption and $R(0)=0$. Therefore $Q, R$, and $f$ stay finite for all finite $\rho$.

From Eq. (3.22) it follows that $0<R^{\prime} \leqslant 1$ for all $\rho \geqslant 0$, so $R=O(\rho)$ for $\rho \rightarrow \infty$. From Eq. (3.21) we get, that $Q$ $=O\left(\rho^{2}\right)$ and from Eq. (3.23) we see that $f^{\prime}$ goes to zero as $\rho^{-3}$ and therefore $f$ goes to a constant at infinity.

(2) $R$ develops an extremum at some finite $\rho_{\text {extr }}>0$. Since $R^{\prime \prime} \leqslant 0$ for all $\rho$ and $R^{\prime}(\rho)<0$ for all $\rho>\rho_{\text {extr }}$, which follows from Eq. (3.22), $R$ goes to zero at some finite $\rho_{S}>\rho_{H}$.

Here we already excluded the case $\rho_{S}<\rho_{H}$ in Sec. III A, since this means a singularity in the static region.

For the same reasons as above, time evolution exists for all $\rho<\rho_{S}$. In the limit $\rho \longrightarrow \rho_{S}$ a spacetime singularity occurs. This follows from inspection of the Kretschmann invariant

$$
\begin{aligned}
R^{\mu \nu \sigma \tau} R_{\mu \nu \sigma \tau}= & \frac{1}{R^{4}}\left[4+4 R^{2} Q^{\prime 2} R^{\prime 2}+R^{4} Q^{\prime \prime 2}\right. \\
& +8 Q R^{\prime}\left(R^{2} Q^{\prime} R^{\prime \prime}-R^{\prime}\right) \\
& \left.+4 Q^{2}\left(R^{\prime 4}+2 R^{2} R^{\prime \prime 2}\right)\right] .
\end{aligned}
$$

Since by assumption $Q(\rho), Q^{\prime}(\rho)$, and $R^{\prime}(\rho)$ are negative near $\rho_{S}$ all terms of Eq. (3.24) are non-negative and at least some of them clearly have a nonzero numerator while the denominator vanishes with some power of $R$.

The construction of solutions beyond the horizon consists of solving the initial value problem, i.e., we integrate Eqs. (3.1)-(3.3) with initial conditions (3.15) for $\rho>\rho_{H}$, where the parameters $\rho_{H}, f\left(\rho_{H}\right), R_{H}$, and $Q_{H}^{\prime}$ are determined by the solutions of the boundary value problem described in Sec. III D. For numerical integration we used routine D02CBF of the NAG library [18].

\section{PHENOMENOLOGY OF SOLUTIONS}

\section{A. Phenomenology of numerically constructed solutions with $\Lambda>0$}

For $\beta=0$ the $\mathrm{E} \sigma_{\mathrm{SU}(2)} \Lambda$ equations decouple into Einstein's vacuum equations with $\Lambda$ and the matter field equation (2.14) on the fixed background. With our regularity conditions at the axis the solution to Einstein's equations is de Sitter space

$$
Q(\rho)=1-\frac{\Lambda \rho^{2}}{3}, \quad R(\rho)=\rho,
$$

whereas the field equation (3.3) admits a discrete one parameter family of regular solutions [4]. Within the static region

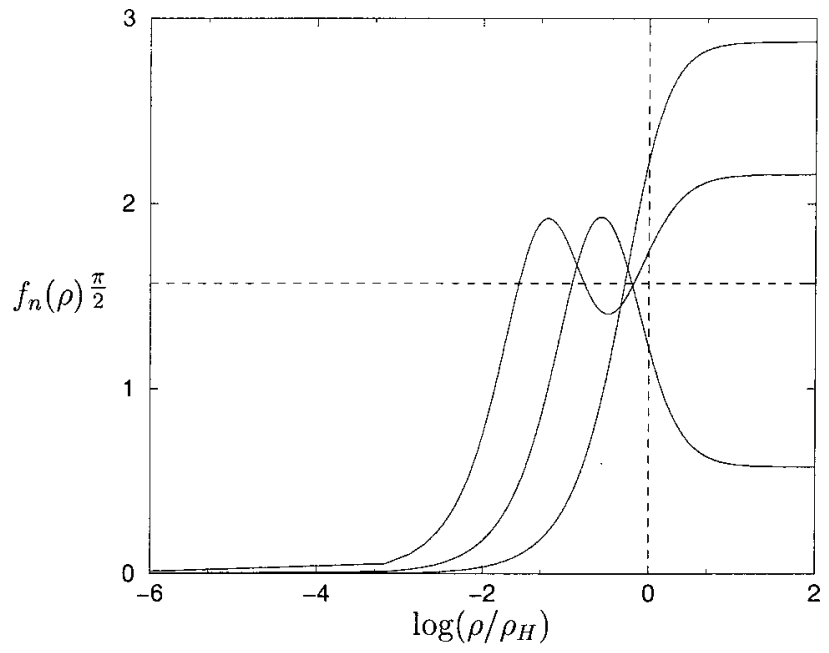

FIG. 1. The first three solutions for coupling constant $\beta=0.3$ $<\beta_{\text {crit }}$. Inside the horizon at $\rho / \rho_{H}=1$ the " $n$ th" excitation crosses the $\pi / 2$ line $n$ times. In the dynamic region outside the horizon the solutions evolve towards a constant.

these solutions oscillate around $\pi / 2$ where energy increases with the number of the oscillations and is limited from above by the energy of the "singular" solution $f \equiv \pi / 2$. Outside the cosmological horizon at $\rho=1$ the solutions remain finite and tend to a constant near infinity.

Increasing the coupling constant $\beta$, the numerical analysis shows, that solutions of this type persist as long as $\beta$ does not get too large. The qualitative behavior of the field $f$ in the static region is the same as in the uncoupled case, i.e., the $n$th excitation oscillates $n$ times around $\pi / 2$, whereas the behavior of the field in the dynamic region as well as the behavior of the geometry depend strongly on the value of the coupling constant $\beta$.

To summarize, we get the following qualitative picture of solutions in dependence on the coupling constant $\beta$.

For small $\beta, 0 \leqslant \beta \leqslant \beta_{\text {crit }}(n)$, the solutions are similar to those of the uncoupled case $\beta=0$, that is, the area of $\operatorname{SO}(3)$ orbits is monotonically increasing with $\rho$, beyond the horizon the solutions persist up to an infinite value of the coordinate time $\rho$. Near infinity the geometry asymptotes to the de Sitter geometry, that is $R=O(\rho)$ and $Q$ tends to $-\infty$ as $O\left(\rho^{2}\right)$. According to Hayward's definitions [2] the horizon is an inner past trapping horizon, separating the static region from an expanding dynamic region. The field $f$ shows the same qualitative behavior as in the uncoupled case $\beta=0$ (see Figs. 1 and 2).

For $\beta=\beta_{\text {crit }}(n)$ the areal radius $R(\rho)$ still increases for all $\rho>0$ but this time goes to a constant at infinity, that is $R^{\prime}(\infty)=0$. For even stronger coupling, $\beta_{\text {crit }}(n)<\beta$ $<\beta_{*}(n), R$ develops a maximum in the dynamic region, i.e., $R^{\prime}\left(\rho_{E}\right)=0$ at some finite time $\rho_{E}>\rho_{H}$ (see Fig. 3). As was discussed in Sec. III E $R$ then decreases and goes to zero at some finite coordinate time $\rho_{S}$-which corresponds to the finite proper time $\tau_{S}=\int_{\rho_{H}}^{\rho_{S}} d \rho / \sqrt{Q(\rho)}$. This causes the geometry to be singular at $\rho_{S}$. The horizon, which again is an inner past trapping horizon separates the static region from 


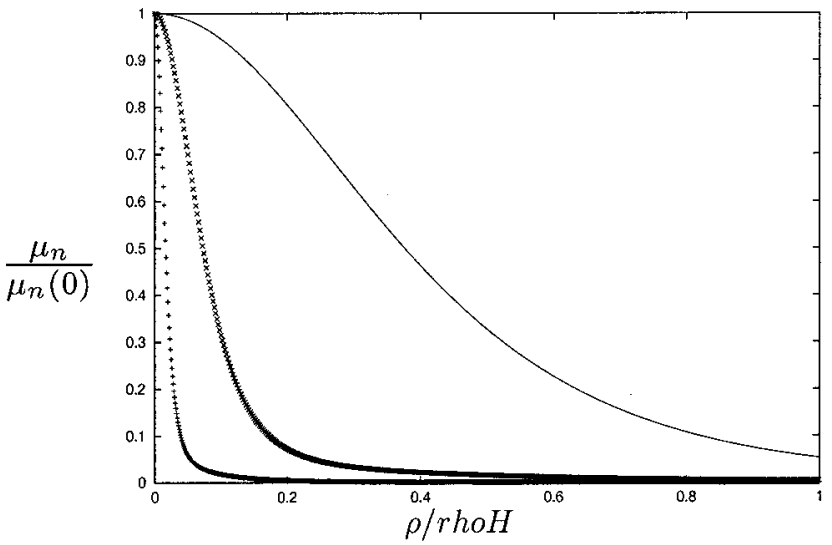

FIG. 2. The energy density $\mu_{n}=Q_{n}\left(f_{n}^{\prime}\right)^{2}+\sin ^{2}\left(f_{n}\right) / R_{n}^{2}$ for the first three excitations at $\beta=0.3$. - denotes the first, $\times$ the second, and + the third excitation, all $\mu_{n}$ have been normalized to unity at the axis. The corresponding total energies, measured in units of $1 / \sqrt{\Lambda}$ are $E_{1} / 4 \pi \gamma=1.66621, E_{2} / 4 \pi \gamma=1.88082$, and $E_{3} / 4 \pi \gamma$ $=1.956613$.

an initially expanding dynamic region, which reaches its maximal spatial extension at $\rho_{E}$ and then recollapses to a singularity at $\rho_{S}$. The maximum of the areal radius occurs at earlier and earlier times as the coupling constant is increased until it merges with the location of the horizon when $\beta$ $=\beta_{*}(n)$.

At $\beta=\beta_{*}(n)$ the "maximal two sphere" coincides with the horizon $\rho_{E}=\rho_{H}$. The fact that $R^{\prime}\left(\rho_{H}\right)=0$ at this value of $\beta$ may be interpreted as exchanging the inward and outward direction at the horizon: for smaller values of $\beta$ the static region was surrounded by the inner past trapping horizon, whereas for larger values of the coupling constant $\beta_{*}(n)<\beta<\beta_{\max }$, where $\rho_{E}<\rho_{H}$, the static region encloses the horizon, which becomes now an outer future trapping horizon. Beyond this horizon, the dynamic region undergoes complete collapse at $\rho=\rho_{S}$.

Embedding diagrams of the static regions of the first excitation for several values of $\beta$ can be found in Fig. 4. We

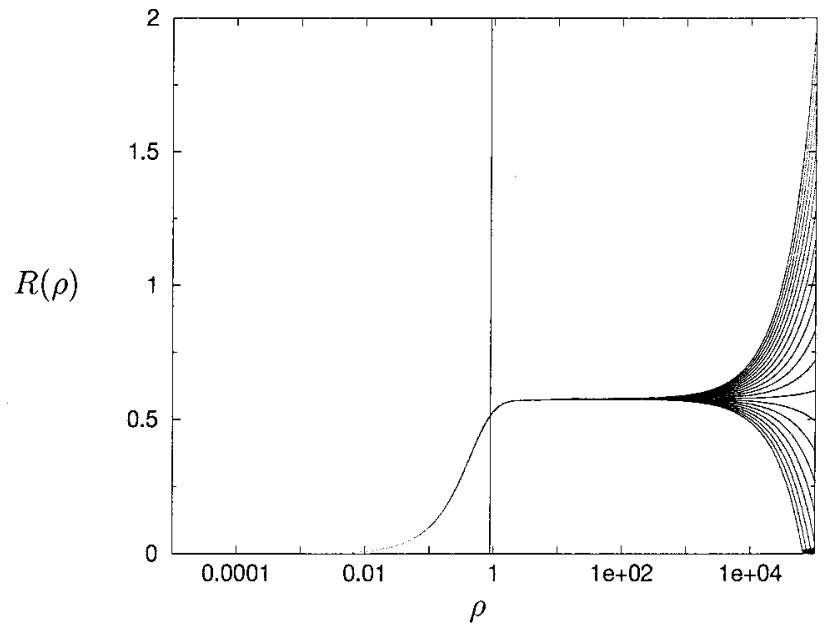

FIG. 3. The area function $R(\rho)$ for the first excitation for $\beta$ near $\beta_{\text {crit }}(1), 0.470366<\beta_{\text {crit }}(1)<0.470373$. The vertical line marks the horizon at $\rho=0.88761$.

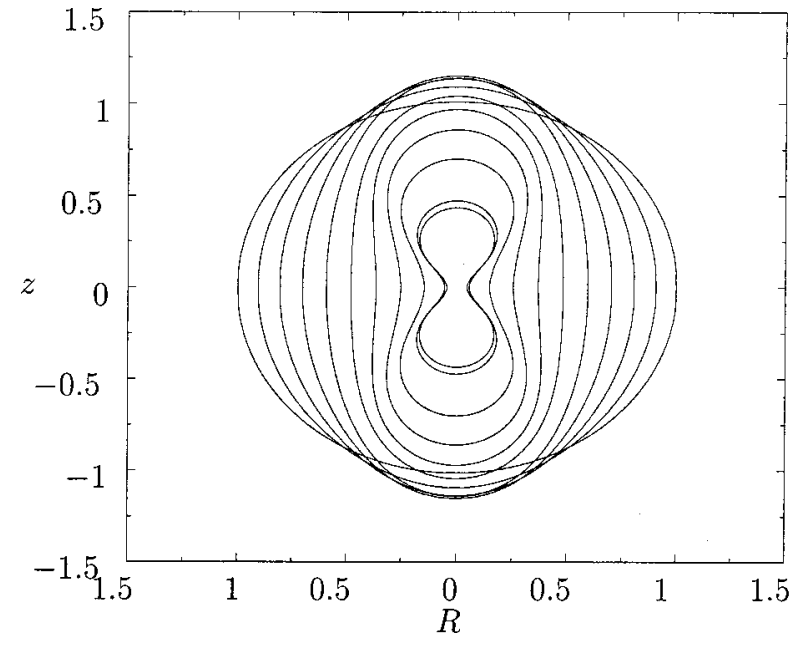

FIG. 4. The rotational surfaces $z(R)$ correspond to the embedding of a $t=$ const slice $(\theta=\pi / 2)$ into $\mathbf{R}^{3}$. The upper and lower half part of the diagram resemble the two static regions causally separated by the horizon at $z=0$. Shown is the geometry of the first excitation for $\beta$ in the range $0 \leqslant \beta \leqslant 0.913<\beta_{\max }$. The sphere of unit radius for de Sitter space $(\beta=0)$ gets more and more deformed as $\beta$ increases.

note, that the "critical" values of the coupling constant, $\beta_{\text {crit }}(n), \beta_{*}(n)$, and $\beta_{\max }(n)$ decrease with the excitation number $n$. In Sec. IV C we will give an argument, that $\beta_{\max }(n)$ is a decreasing sequence, which is bounded from below by the maximal coupling constant $\beta_{\max }(\infty)=1 / 2$ for the "singular" solution (3.6).

The solutions described above exist in the presence of a positive cosmological constant $\Lambda>0$. As will be described in detail in the next section, Sec. IV B the limit $\beta \rightarrow \beta_{\max }$ yields regular solutions iff one takes the limit $\Lambda \rightarrow 0$ appropriately as described in Sec. IV B.

\section{B. The limit $\beta \rightarrow \beta_{\max }(n)$}

Recall from Sec. III A that the cosmological constant $\Lambda$ sets the length scale in Eqs. (3.1)-(3.4) and that it can be eliminated from these equations, by introducing the dimensionless quantities $\bar{\rho}=\sqrt{\Lambda} \rho$ and $\bar{R}=\sqrt{\Lambda} R$. This corresponds to measuring all quantities that have dimension of length, such as, e.g., the energy $E$, the coordinate distance of the horizon $\rho_{H}$ from the origin, the radial geometrical distance of the horizon $d_{H}$ from the origin, the areal radius $R_{H}$ of the horizon, and $1 / f^{\prime}(0)$, in units of $1 / \sqrt{\Lambda}$. We find that all parameters, that have dimension of length go to zero in the limit $\beta \rightarrow \beta_{\max }$ when measured with respect to this length scale. This indicates that $1 / \sqrt{\Lambda}$ is not the appropriate length scale for taking this limit. We therefore switch to the alternative viewpoint of $\rho_{H}$ as our length scale, and we fix $\rho_{H}$ $=1$. In this setup $\Lambda$ depends on $\beta$ and the excitation index $n$ and goes to zero in the limit $\beta \rightarrow \beta_{\max }$. The parameters $E$, $d_{H}$, and $1 / f^{\prime}(0)$ attain finite values when measured in units of $\rho_{H}$, whereas $R_{H} / \rho_{H}$ goes to zero (see Fig. 5). This strongly suggests that there exists a solution with $\beta=\beta_{\max }$ which obeys Eqs. (3.1)-(3.4) with $\Lambda=0$ and has two centers 


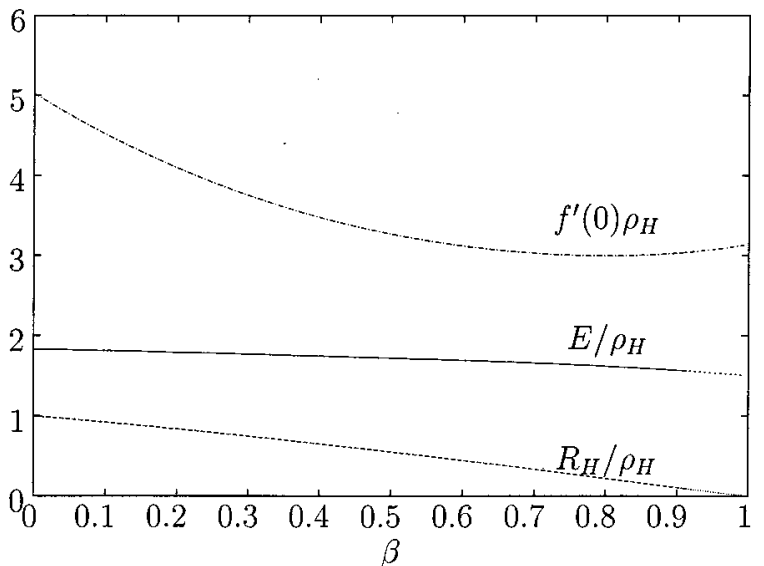

FIG. 5. Some parameters with dimension length measured in units of $\rho_{H}$. Except $R_{H}$ all of them stay finite in the limit $\beta$ $\rightarrow \beta_{\max }$. Moreover in the limit the values tend to the corresponding ones of the static Einstein universe, as given in Table I.

of symmetry. In particular this means that the static region of this solution has no boundary, since any $t=$ const slice has topology $\mathbf{S}^{3}$.

Furthermore, as can be seen from Fig. 6, the dimensionless parameter $f\left(\rho_{H}\right)$ for the first excitation tends to $\pi$, and $R^{\prime}\left(\rho_{H}\right)$ tends to -1 in the limit $\beta \rightarrow \beta_{\max }$. As will be shown in the next section, Sec. IV C, $\Lambda=0$ implies $Q \equiv 1$. The limiting solution with $\Lambda=0$ will therefore satisfy the regularity conditions (2.9) and (2.15) not only at the axis $\rho=0$ but also at the second zero of $R$, which means that such a solution is globally regular with two (regular) centers of spherical symmetry. In fact, for the first excitation this limiting solution is just the static Einstein universe (3.7), which can be given in closed form.

These observations allow one to determine the maximal value of the coupling constant $\beta_{\max }(n)$ not as a limiting procedure $\beta \rightarrow \beta_{\max }$, but rather by solving the boundary value problem Eqs. (3.1)-(3.3) with $\Lambda=0$ and with boundary conditions, that correspond to two regular centers of symmetry.

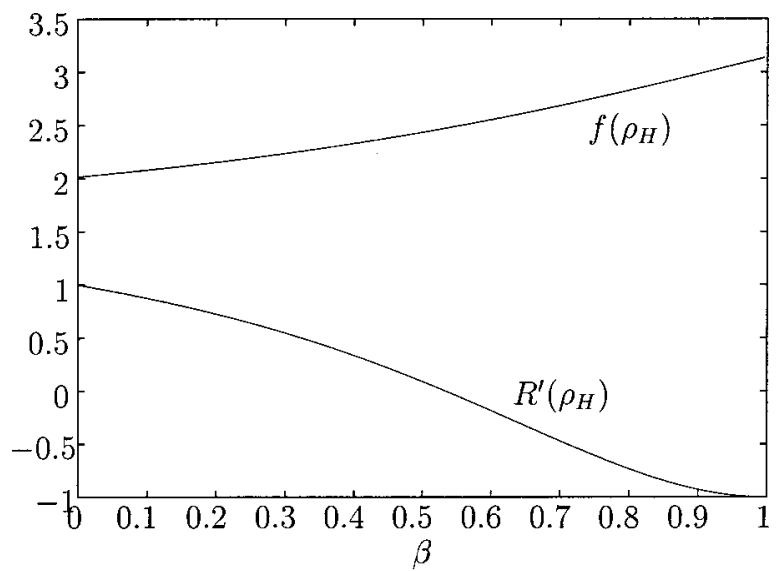

FIG. 6. The (dimensionless) parameters $f\left(\rho_{H}\right) \equiv b$ and $R^{\prime}\left(\rho_{H}\right)$ of the first excitation in dependence of the coupling constant $\beta$. $f\left(\rho_{H}\right)$ tends to $\pi$ in the limit $\beta \rightarrow \beta_{\max }$ and $R^{\prime}\left(\rho_{H}\right)$ tends to -1 in this limit.

\section{Globally static, regular solutions for $\Lambda=0$}

For $\Lambda=0$ Eq. (3.1) can be solved immediately to give $R^{2} Q^{\prime}=$ const. According to the regularity conditions at the axis (2.9) the constant has to vanish, which means that $Q^{\prime}$ $\equiv 0$ and therefore $Q \equiv 1$. The remaining system of equations is

$$
\begin{aligned}
R^{\prime \prime} & =-\beta R f^{\prime 2}, \\
\left(R^{2} f^{\prime}\right)^{\prime} & =\sin (2 f),
\end{aligned}
$$

and

$$
2 \beta \sin ^{2} f-\beta R^{2} f^{\prime 2}+R^{\prime 2}-1=0 .
$$

Note, that this system of ODEs is scale invariant, that is any solution $R(\rho), f(\rho)$ leads via rescaling to the one parameter family of solutions given by $a R(a \rho), f(a \rho)$. Keeping this in mind, we can fix the scale arbitrarily, e.g., in setting the first derivative of the field $f$ equal to one at the origin: $f^{\prime}(\rho=0)$ $=1$. Thereby any solution, that is regular at the origin, is determined entirely by the value of the coupling constant $\beta$.

Regularity conditions at the second "pole" $R\left(\rho_{P}\right)=0$ are the same as at the origin, except that $f$ either tends to $\pi$, if its excitation number is odd, or to 0 if it has even excitation number. This can be inferred from $\pi / 2<f\left(\rho_{H}\right)<\pi$ for $n$ odd and $0<f\left(\rho_{H}\right)<\pi / 2$ for $n$ even for all $\beta<\beta_{\max }$, since this is the case for $\beta=0$ and according to Eq. (3.15) no crossing of the zeroline or $\pi$ line is allowed. Note that this corresponds to all odd solutions having winding number 1 , whereas even solutions are in the topologically trivial sector.

These regularity conditions together with the invariance of the equations under reflection at the location of the maximal two sphere $R^{\prime}\left(\rho_{E}\right)=0$, causes globally regular solutions $R(\rho)$ to be symmetric around $\rho_{E}$ whereas $f(\rho)-\pi / 2$ is either antisymmetric for $n$ odd or symmetric for $n$ even.

For $f$ symmetric the formal power series expansions of $R(\rho)$ and $f(\rho)$ around $\rho=\rho_{E}$ gives

$$
\begin{aligned}
R(\rho)= & R\left(\rho_{E}\right)+O\left[\left(\rho-\rho_{E}\right)^{4}\right], \\
f(\rho)= & \arcsin \sqrt{1 / 2 \beta}+\frac{2 \sqrt{1-1 / 2 \beta}}{R\left(\rho_{E}\right)^{2} \sqrt{2 \beta}} \frac{\left(\rho-\rho_{E}\right)^{2}}{2 !} \\
& +O\left[\left(\rho-\rho_{E}\right)^{4}\right],
\end{aligned}
$$

and for $f-\pi / 2$ antisymmetric we get

$$
\begin{aligned}
& R(\rho)=\frac{2 \beta-1}{\beta f^{\prime}\left(\rho_{E}\right)^{2}}-(2 \beta-1) \frac{\left(\rho-\rho_{E}\right)^{2}}{2 !}+O\left[\left(\rho-\rho_{E}\right)^{4}\right] \\
& f(\rho)=\frac{\pi}{2}+f^{\prime}\left(\rho_{E}\right)\left(\rho-\rho_{E}\right)+O\left[\left(\rho-\rho_{E}\right)^{3}\right]
\end{aligned}
$$

In order to solve the system (4.2), (4.3) we again use the shooting and matching method on the interval [origin, $\rho_{E}$ ] using the above Taylor series expansions to determine the boundary conditions at $\rho=\rho_{E}$. Shooting parameters are now 
TABLE I. Results for the first three excitations for $\Lambda=0$. Since $Q \equiv 1$ the coordinate distance $\rho_{P}$ of the two regular "poles", equals the radial geometrical distance $d_{P}$. The energy density $\rho_{P}$ and energy $E$ are given in units where $f^{\prime}(0)=1$. The ratio $E / d_{P}$ can be compared to the results for solutions with $\Lambda>0$ and represents the limit $\beta \rightarrow \beta_{\max }$ for those solutions.

\begin{tabular}{ccccc}
\hline \hline$n$ & $\beta_{\max }$ & $\rho_{P}=d_{P}$ & $E / 4 \pi \gamma$ & $E / 4 \pi \gamma d_{P}$ \\
\hline 1 & 1 & $\pi$ & $3 \pi / 2$ & $3 / 2$ \\
2 & 0.74255 & 6.74225 & 11.78039 & 1.74724 \\
3 & 0.64931 & 12.10140 & 22.43662 & 1.85405 \\
\hline \hline
\end{tabular}

$\rho_{E}, f^{\prime}\left(\rho_{E}\right)$, and $\beta$ for odd solutions and $\rho_{E}, R\left(\rho_{E}\right)$, and $\beta$ for even solutions. The results are displayed in Table I.

It is clear from Eqs. (4.5) and (4.6), that regular solutions for $\Lambda=0$ can only exist if $\beta>1 / 2$. Assuming now, that our numerical observations concerning the first few excitations extend to higher excitations, we give the following argument. Since every "branch" of the " $\Lambda>0$ solutions", persists up to a maximal value of beta, which can be computed by solving the boundary value problem (4.2) together with regularity conditions at the two "poles" - which implies $\beta$ $>1 / 2$ - and since we know, that in the limit $\beta \rightarrow 0$ there exists an infinite number of excitations [4], we conclude that this whole family of solutions with $\Lambda>0$ persists up to some maximal value $\beta_{\max }(n)$, which is greater than $1 / 2$. In other words, for any $\beta<1 / 2$ there exists a countably infinite family of solutions with $\Lambda>0$, whereas for $\beta>1 / 2$ our numerical analysis shows, that only a finite number of solutions exists (see Table I).

\section{DISCUSSION AND OUTLOOK}

We have shown numerically that the $\mathrm{SU}(2)-\sigma$ model coupled to gravity with a positive cosmological constant admits a discrete one-parameter family of static spherically symmetric regular solutions. These solitonic solutions are characterized by an integer excitation number $n$. A given excitation will only exist up to a critical value of the coupling constant $\beta$; the higher $n$, the lower the corresponding critical value. Our calculations indicate that the infinite tower of solitons present on a de Sitter background persists at least up to a value of $\beta=1 / 2$. Thus there exists a $\beta \geqslant 1 / 2$ beyond which the number of excitations is finite and decreases with the strength of the coupling. As mentioned, qualitatively the $\sigma$-model under consideration shows striking similarities to the EYM system as studied in detail by Volkov et al. The main difference being that the static solutions to the EYM system depend on the value of the cosmological constant while in our case $\Lambda$ scales out from the equations and $\beta$ plays the role of a "bifurcation" parameter. Another difference concerns the globally regular static solutions with compact spatial slices. For the EYM system these appear for definite values of $\Lambda(n)$ while for the $\sigma$ model the corresponding solutions exist only in the (singular) limit as $\Lambda$ goes to zero and definite values of $\beta$. Thus in our case there are closed static universes with vanishing cosmological constant, the lowest excitation being the static Einstein cosmos. This is possible because in this case the stress-energy tensor of the $\sigma$ field is of the form of a perfect fluid with the equation of state $p=-\mu / 3$. Another interesting aspect is the geometry of a given excitation as a function of the coupling strength: the static region is always surrounded by a Killing horizon separating the static from a dynamical region, which for small couplings becomes asymptotically de Sitter. As the coupling is increased the two spheres of symmetry beyond the horizon are first past and then become future trapped and a cosmological singularity develops. Finally, for even stronger couplings, again the region beyond the horizon collapses, but within the static region the ingoing and outgoing directions (as defined by the sign of the expansion for null geodesics) interchange.

An important question to be answered is whether these solitons are stable under small radially symmetric timedependent perturbations. In a forthcoming publication we intend to present a detailed stability analysis. We will show that for $\Lambda>0$ all excitations are unstable with their number of unstable modes increasing with $n$. This was to be expected at least for small coupling. The lowest excitation thus has a single unstable mode and it is known, from other models, that such a solution can play the role of a critical solution in a full dynamical treatment of spherically symmetric collapse.

\section{ACKNOWLEDGMENTS}

We thank J. Thornburg and M. Pürrer for computer assistance and especially P. Bizon for helpful comments and his interest in this work. This research was supported in part by FWF as Project No. P12754-PHY and the Fundacion Federico.
[1] M. S. Volkov, N. Straumann, G. Lavrelashvili, M. Heusler, and O. Brodbeck, Phys. Rev. D 54, 7243 (1996).

[2] S. A. Hayward, Phys. Rev. D 49, 6467 (1994).

[3] P. Bizon, Proc. R. Soc. London A451, 779 (1995).

[4] P. C. Aichelburg and C. Lechner, Phys. Rev. D 57, 6176 (1998).

[5] R. Bartnik and J. McKinnon, Phys. Rev. Lett. 61, 141 (1988).

[6] N. Straumann and Z. H. Zou, Phys. Lett. B 237, 353 (1990).

[7] M. W. Choptuik, T. Chmaj, and P. Bizon, Phys. Rev. Lett. 77, 424 (1996).

[8] M. W. Choptuik, in Gravitation and Relativity: At the turn of the Millennium, Proceedings of the GR-15 conference, edited by N. Dadhich and J. Narlikar, Inter-University Centre for Astronomy and Astrophysics, Pune, India, 1998.

[9] M. Heusler, Helv. Phys. Acta 69, 501 (1996).

[10] C. Lechner, S. Husa, and P. C. Aichelburg (in preparation).

[11] M. Heusler, Black Hole Uniqueness Theorems, Cambridge Lecture Notes in Physics No. 6 (Cambridge University Press, Cambridge, England, 1996).

[12] C. W. Misner, Phys. Rev. D 18, 4510 (1978). In particular Misner points out that harmonic maps are, at a more fundamental level, geometrically natural nonlinear theories, (as are 
gravity and Yang-Mills theory) that in a sense combine the nonlinearity of the geodesic equation with the propagation features of the wave equation, which are both contained as special cases.

[13] G. W. Gibbons, in The Physical Universe, Lecture Notes in Physics No. 383, edited by J. Barrow (Springer, Berlin, 1991).

[14] For a recent overview on the topic of gravitating particle-like and black hole solutions of nonlinear matter fields see M. S. Volkov and D. V. Gal'tsov, Phys. Rep. 319, 1 (1999).
[15] S. W. Hawking and G. F. R. Ellis, The Large Scale Structure of Spacetime (Cambridge University Press, Cambridge, England, 1973).

[16] S. A. Hayward, Phys. Rev. D 53, 1938 (1996).

[17] R. Penrose, in Battelle Rencontres, edited by C. M. DeWitt and J. A. Wheeler (Benjamin, New York, 1968).

[18] NAG FORTRAN Library Manual-Mark 16, The Numerical Algorithms Group, Ltd., Oxford, 1994. 